\title{
Study on the Development of College Students' Psychological Health
}

\author{
Yao $\mathrm{Wu}^{1}$, Lian Huang ${ }^{1}$ \\ ${ }^{1}$ Chengdu University of Information Technology, Chengdu, Sichuan, 610100
}

Keywords: College Student, Psychological Health, Development Study

\begin{abstract}
The psychological health education for college students in China began in the mid-1980s and has now entered a period of rapid development. In particular, the "Some Opinions of the Central Committee of the Communist Party of China on Further Strengthening and Improving School Moral Education Work" (1994) have been issued. The mental health education of college students has received much attention, and was incorporated into the work plan of college and university. At present, the mental health education of college students has shown a vigorous development trend. In recent years, due to the extensive and in-depth study of the psychological health of college students, exciting achievements have been made, which is of great importance for the further development of mental health education in colleges and universities.
\end{abstract}

\section{Introduction}

Mental health has a profound impact on the growth of college students. This fast-paced life in modern society has aggravated people's psychological burden, especially when they are young. Their psychological development is in the transitional stage from immature to maturity. Therefore, the psychological health of college students has become an increasingly pressing issue for social development. College students' academic pressure, love, and interpersonal communication will affect their mental health. A large number of college students Because one of life's unsatisfactory results puts himself in a dead end. What are the factors that affect the mental health of college students? This article summarizes some of the experimenter's research results, systematically discusses the status quo of college students' psychological health and the influencing factors, and looks forward to the study of college students' mental health. Taking a survey of previous studies on the psychological health of college students, many researchers use questionnaires to conduct research.

\section{The Current Situation and Influencing Factors of College Students' Mental Health}

The Ma Jiajue incident and a series of incidents caused by mental health problems have aroused the attention of the society. A large number of investigations and studies have shown that the level of mental health of college students is far lower than the average level of the national youth.

There are many factors that affect the mental health of college students. The family factor as a basic factor has a profound effect on the psychological health of college students. For example, Millikan and others used the Eisenke Personality Questionnaire to find that the role of the family has a significant relationship with depression in college students. Parents play an important role in the process of socialization of their children. However, due to factors such as the popularization of electronic communication equipment, the connection between college students and parents is still very tight, and university students have been unable to do "psychological weaning". First of all, college students' parenting styles are important factors affecting their mental health. Different ways of rearing will have different effects. Parental rearing methods are divided into three types: authoritative (strong parents, enthusiastic, and taking into account the child's opinion), autocratic type (regardless of the child's requirements), and beloved type (incapable parents allow children to do whatever they like). Studies have shown that the level of mental health of college students in a democratic education is higher than that of an arbitrarily typed education (Lin Sibao, Schumann, 2003). If the parents fully understand the child and give warmth, it will have a positive impact on 
the child's mental health [1]. If parents interfere too much with children, refusing to deny them will have a negative impact on the child's mental health. Second, the family structure will also affect the psychological health of college students. Research shows that the number of children in the family has a significant impact on the psychological health of college students. The overall level of mental health of college students with siblings of 2 or 3 is significantly worse than that of only children. And the marital status of parents has a significant impact on the mental health of college students. Parental divorce is worse than the normal level of mental health of normal college students, and parents divorced are more hostile than the college students whose parents died. In addition, family economic status, parental education, parental career, family relationships, family atmosphere, and parents' expectations of their children will also affect the mental health of college students.

In addition to being influenced by family factors, the mental health of college students is also influenced by personal factors. During this period, the self-evaluation of college students has a halo effect. Young people who become college students generally experience many successes and have received many praises [2]. Therefore, their awareness of their abilities is often high. If in actual life, some of the surrounding objective evaluations are inconsistent with their self-recognition, they will produce negative emotions and cause psychological imbalances. Second, the personality of college students is a decisive factor in mental health. The college period is a period of both desire for friendship and the pursuit of loneliness. Different personality college students have different mental abilities. A person with a dissociated personality and who does not like to socialize with others is likely to be mentally unbalanced when faced with setbacks. If it goes on like this for a long time, it will induce mental illness. In addition, college students' learning and life are tense. The pressure of learning can easily lead to negative emotions. In addition, college students' emotions are in a more complex period. The ability to regulate and control emotions is relatively weak. If the external environment changes, the individual's psychology will change. affected. To reach the level of mental health, we must have a stable mood and love life.

\section{Problems in the Research of College Students' Mental Health}

Judging from the current research, because the criteria for judging the mental health of college students are not uniform, that is, the mental health in the concept of the researcher is not the same structure, different research results emerge. Most researchers did not define the mental health standards of college students when they conducted mental health surveys and assessments. Most of the reference was based on ordinary people's mental health standards. There is also a different understanding of the mental health standards of the average person, and therefore there is controversy over survival standards and development standards. Scholars in our country are more inclined to survival standards (or social adaptation standards). For example, most scholars' mental health standards mostly reflect only the general well-adapted psychological state of an individual's social life. Requirements [3]. In other words, most of the college students' mental health standards are still in a narrow sense. They focus on the study of college students' psychological problems and psychological problems, and ignore the positive aspects of the university students' mental health, especially paying less attention to their potential and personality. . College students are a special group. College mental health problems are both common to common people's mental health and the particularity of this group. In the investigation of college students' psychological health, if they apply general standards blindly, and do not consider their physical growth, physiological specificity, academic burden, achievement motivation, aspiration level, life orientation, personality shaping and other development factors, there will be problems of unreliable research results or inability to make comparisons between research results due to inconsistent criteria.

Judging from the relevant data, China's current empirical research on the psychological health of college students is mostly based on foreign ready-made scales for simple investigation or general verification. Some researchers have used the mental health research articles published in the Chinese Mental Health Journal as an example to analyze that $61.5 \%$ of articles are assessment studies or comparative studies of the mental health status of different types of college students, of which mental health self-assessments. Scale (SCL -90) is the most used scale, followed by Cattell 
16 Personality Rating Scale (16PF), Eisenk Personality Scale (EPQ), Zung's Anxiety Scale (SAS), Life Questionnaires for incidents [4]. Through comparative analysis, the scope and limitations of these scales and questionnaires can be seen. SCL-90 (Symptom Checklist) measures the level of symptoms or mental status of a person over a period of time, including psychotic disorders, involving feelings, thinking, emotions, behavior, consciousness, interpersonal relationships, lifestyle habits, diet, the 90 items in many aspects such as sleep are mainly used in clinical research, psychological counseling, psychiatry, and outpatient services to assess whether a person has certain psychological symptoms and how serious they are. This scale is a standard reference test and uses psychological symptoms as a criterion to judge whether a person has certain psychological symptoms and severity.

\section{Suggestions on Research of College Students' Mental Health}

Mental health includes two parts that are closely related to each other and different from each other in terms of psychological state and psychological adjustment ability. Mental state is the state of one's self-perceived mental state (especially good or bad mood) at a certain time or during a certain period of time. It is susceptible to life events. Psychological adjustment ability is a person's ability to adjust his or her mental state to an appropriate level. This ability can be improved through learning and exercise. In addition, mental health also has a narrow and broad sense. The narrow sense of mental health mainly refers to the prevention of mental disorders or behavioral problems; the broad sense of mental health aims to promote people's psychological adjustment and develop greater mental performance, that is, to enable people to live healthy lives in the environment and keep improving. The level of mental health, so as to better adapt to social life, contribute more effectively to society and human beings. In the study of college students' mental health, it is necessary to study the psychological diseases, psychological barriers and psychological problems of college students. However, this is still not enough. The problem of college students' mental health should also be explored from the developmental aspects of the college students. In particular, quality education, which focuses on cultivating students' creative spirit and practical ability, puts forward new and higher requirements for the cultivation of talents. Students are faced with a broader world where they can display their talents, their independence, creativity, the components of developmental and other qualities must be reflected when assessing mental health. Through the study of the psychological characteristics of college students and through appropriate evaluation methods, it can be used to mental health education as soon as possible to indicate the direction of psychological development, so that it not only has a higher level of professional knowledge, but also has a perfect personality and is of great significance [5].

Should study and explore effective psychological health assessment tools for college students. From the existing research, many theories of psychology have a lot of descriptions of mental health from a certain perspective or on a certain level, but these descriptions have not been transformed into effective evaluation tools through empirical research. Mental health standards still remain on the theoretical controversy, and empirical and localized assessment tools are rare. Research and development of questionnaires that are in line with the actual psychological health of contemporary Chinese college students should be a top priority for researchers. This will be of some significance. It provides a basis for the evaluation and research of college students' mental health.

With the development of modern medicine, physics, biology, and information technology, it has laid a solid scientific foundation for the psychological health education of college students and provided modern education methods. For example, in the psychological training of college students, more emphasis will be placed on "physical-psychological training methods" and "biological-psychological training methods", with the help of modern instruments and equipment to scientifically and effectively carry out mental health education for college students; psychological problems detection and psychological file construction for undergraduates. In the past, a large number of modern information technologies were used to make the test results scientific. In the process of psychological counseling and psychological counseling for college students, not only manual methods (such as recording, conversation, etc.) were used, but also rely heavily on 
computers and information networks, online psychological schools and Psychological counseling agencies will be greatly developed. The opening of the website of the Chinese Mental Health Association's Psychological Consultation Committee's Xinqiao website, the Psychedelic Chinese Psychology website for psychological education, and the "China Education Fund" of the China Institute of Health Education mark the entry of China's mental health education into the Internet. era. Modern science and technology will enable the popularization of mental health education among college students and will greatly improve the performance of mental health education.

\section{Conclusion}

On the basis of the relevant literature research, this article analyzes the problems in the study of college students' mental health, and considers that there are inconsistent standards in the study of the mental health of college students, the evaluation measures are mostly at the level of diseases and disorders, and the measurement tools lack effectiveness, insufficient localization and other issues. Then we put forward the evaluation of the psychological health of college students must first pay attention to the evaluation criteria, and use an empirical method to explore the questionnaire in line with the actual psychological health of college students.

\section{References}

[1] He Rui. Research on the influence of college students' sports lifestyle on mental health. Social Sports. 22(5):187-188.

[2] Ling Sibao, SHU Man. Investigation and analysis of the influence of family factors on the personality of college students. Journal of East China Jiaotong University. 20(3): 91-94.

[3] Zeng Meiying, Yu Ning, Yu Hongjun, Lu Danlei. Research on the influence of family factors on mental health of college students. Psychological Science. 31(3): 597-601.

[4] Cheng Suping, Zhang Chao, Jia Jianrong. Influence of social support on mental health of college students. Chinese Journal of Health Psychology. 17(1):35-37.

[5] Yin Hengxi, Lu Min, Wang Xinli, Chen Yanfei. Research on the Influence of Sports on the Mental Health of College Students. Sports Science. 27(5):41-47. 